\title{
New Operational Availability Model to Evaluate Manufacturing Throughput: Advanced TPS for Global Production
}

\author{
Hirohisa Sakai ${ }^{1, *}$, Joe Li $^{2}$ \\ ${ }^{1}$ Toyota Motor Corporation, 1, Motomachi, Toyota-shi, Aichi-ken, Japan \\ ${ }^{2}$ Toyota Motor Manufacturing, Texas, Inc., 1 Lone Star Pass, San Antonio, TX 78264, USA
}

Received January 12, 2020 ; Revised February 21, 2020; Accepted February 24, 2020

Copyright $\odot 2020$ by authors, all rights reserved. Authors agree that this article remains permanently open access under the terms of the Creative Commons Attribution License 4.0 International License

\begin{abstract}
To achieve simultaneous, worldwide high quality assurance and other global production developments, today's task is to maintain high reliability in production facilities and operations. In response to the increasing expansion of overseas plants, it is necessary to improve and maintain highly accurate production equipment and operations through the development of intelligence operators. The authors have clarified Advanced TPS as a global production technology and management model designed to realize high quality assurance in global production. Manufacturing throughput (Throughput) is oriented toward the reduction of time required by the manufacturing process, so that the profitability can be increased. Therefore, the authors have recognized the operational availability a key indicator in order to evaluate the throughput. This paper will introduce a new operational availability model to identify 1) the development of throughput to link the impact from one step to following step and 2) the contribution of each factors and each step for manufacturing process to pinpoint the bottleneck of the throughput. With improvement to these bottlenecks, the business will be successful while minimizing cost. The effectiveness of this system has been verified at the Toyota USA.
\end{abstract}

Keywords Operational Availability, Advanced TPS, Throughput, Toyota USA

\section{Introduction}

Japanese production management system methodologies that contributed to global business in the latter half of the $20^{\text {th }}$ century can be represented by the traditional Toyota Production System that constitutes the foundation of JIT (Just in time). In recent years, Japanese manufacturers are endeavoring to survive in a competitive market by expanding their global production and achieving worldwide uniform quality and simultaneous new model launches. Given this background, the authors have recognized the necessity for advancement of Japanese production management system methodologies and therefore hereby propose "Advanced TPS[1]".

Manufacturing throughput (Throughput) is known for the amount of time required for a product to pass through a manufacturing process $[2,3]$. The concept of throughput is primary oriented toward the reduction of time required by the manufacturing process $[4,5]$. However, it cannot be seen the article about the processing capacity improvement in consideration of the work delay, maintenance of facilities and the influence of sequential system aspect each other. So the authors have recognized the operational availability a key indicator in order to evaluate the throughput and proposed a new operational availability model to identify 1) the development of throughput to link the impact from one step to following step and 2) the contribution of each factors and each step for manufacturing process to pinpoint the bottleneck of the throughput. With improvement to these bottlenecks, the business will be successful while minimizing cost.

This involves the systematization of Japanese production management methodologies as a strategic tool for global production. The authors later demonstrated the new production operational availability model of the proposed "Advanced TPS" at the Toyota in the USA (Toyota USA).

\section{Background}

\subsection{Traditional Operational Availability Model}

Toyota Motor Corporation (Toyota) is known for setting 
the standard in manufacturing industries for producing products in the fastest and most efficient way because of the Toyota Production System (TPS) [6-8]. When Toyota builds a plant in Japan or abroad, TPS has been implemented to optimize the production process. It has successfully reduced manufacturing lead times, reduced work in process, and improved work environment and manufacturing operation.

On the other hands, the throughput is primary oriented toward the reduction of time required by the manufacturing process, so that the profitability can be increased. With TPS, the throughput of each step is closely connected together because of reduced inventory. A primary objective of TPS is to achieve smooth continuous flow. It is very important to identify and improve the throughput bottleneck step to improve TPS operation flow.

One main metrics of throughput evaluation is operational availability (OA). In TPS, the operational availability is defined as the probability that a system is ready to perform its mission or how well the product and process satisfies end user demands.

In traditional operational availability model, the overall throughput of a system is calculated at last step (step 4 as shown in Figure 1) $[9,10]$. However, it cannot be seen the article about the processing capacity improvement in consideration of the sequential system aspect each other.

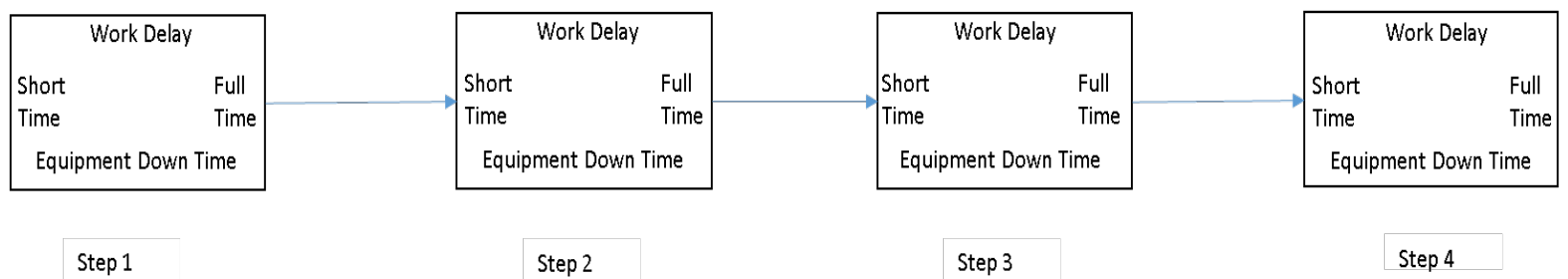

Figure 1. Traditional Operational Availability Model

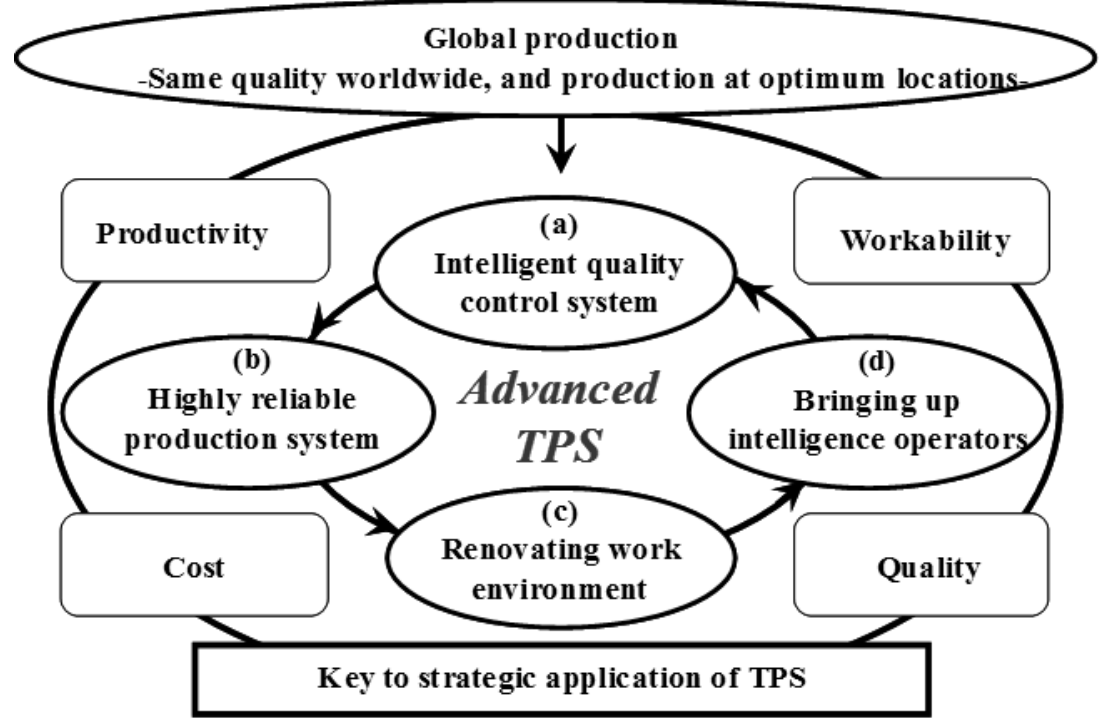

Figure 2. New Japanese Production System Model, Advanced TPS 


\subsection{Necessity of New Operational Availability Model}

This operational availability model, as shown in Equation (1), can also be applied to evaluate each step operation status. Although the OA is a good indicator to show the throughput of the plant or even each step, it is difficult to analyze the bottleneck or to understand the reason behind the $\mathrm{OA}$ issues. The throughput can be affected by equipment or production issue, furthermore, short time (starvation) and full time (blockage) and other reasons. It is easy to track issues at each step but hard to judge the impact through each step to overall throughput.

The importance of quantifying elements for the manufacturing plant is to find areas for improvement. For example, if the overall OA is $92 \%$ and the OA target is $98 \%$ then the gap is $6 \%$. If we know the $90 \%$ OA gap is due to equipment and $10 \%$ OA gap is due to production repair, the opportunity to close the OA gap for improving equipment is usually much greater than for improving production repair.

In order to accomplish TPS smooth floor, the authors have proposed a new production operational availability model to evaluate production throughput to pinpoint where to make improvements in consideration of the work delay, maintenance of facilities and the influence of sequential system aspect each other. It also helps to standardize the throughput evaluation process for global production.

$O A=$ Production Time-Short Time-Equipment Time-Work Delay Time Production Time

$100 \%$

Given this background, the authors have recognized the necessity for advancement of Japanese production management system methodologies and therefore hereby propose "Advanced TPS" [11-14] as shown in Fig. 2. Advanced TPS is meant as a methodology to attain high quality assurance in global production. Advanced TPS is comprised of four different pillars: Productivity, Cost, Workability, and Quality. This proposal focuses on the pillar of productivity by implementing a system which will be able to improve and maintain efficient inventory management throughout the plant. Today we will describe the inventory management subject which is mainly in relation with the cost and productivity pillars of Advanced TPS.

\section{Proposal: New Operational Availability Model for Advanced TPS}

\subsection{Strategic Development of the New Operational Availability Model}

In TPS, the inventory buffer between each step is low so the overall impact of all previous steps affects the final throughput through short time (manufacturing starvation) to following step. Each step also affects the throughput of previous steps through full time (manufacturing blockage). The impact of each step or certain type of downtime can be evaluated by analyzing relationships between the so-called manufacturing blockage (Full) and manufacturing starvation (Short) of each step, as shown in Fig. 3.

\subsection{Proposal of Advanced TPS for Global Production}

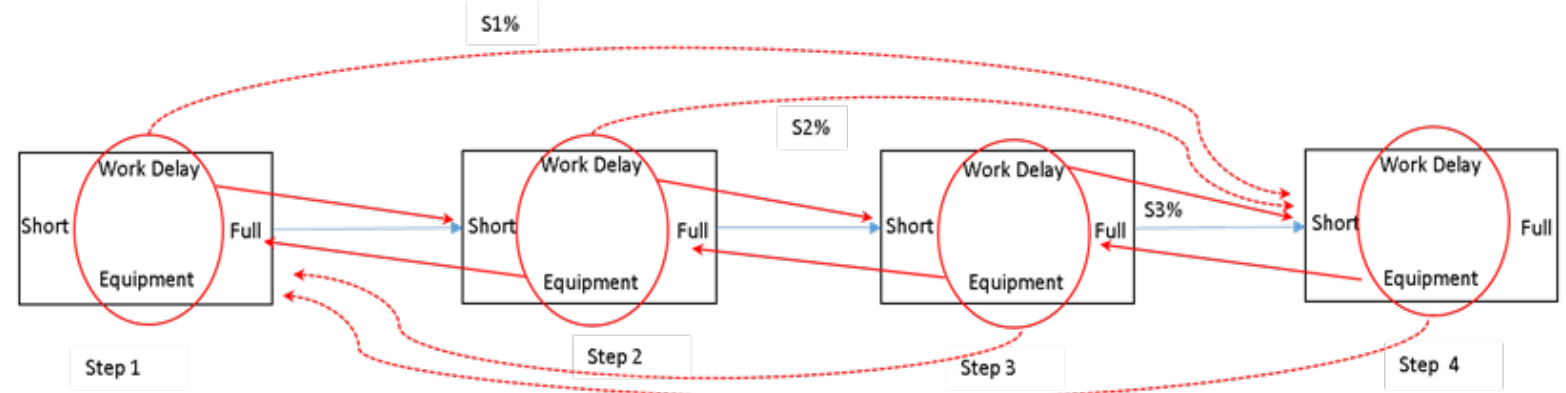

Figure 3. Proposed Operational Availability Model 


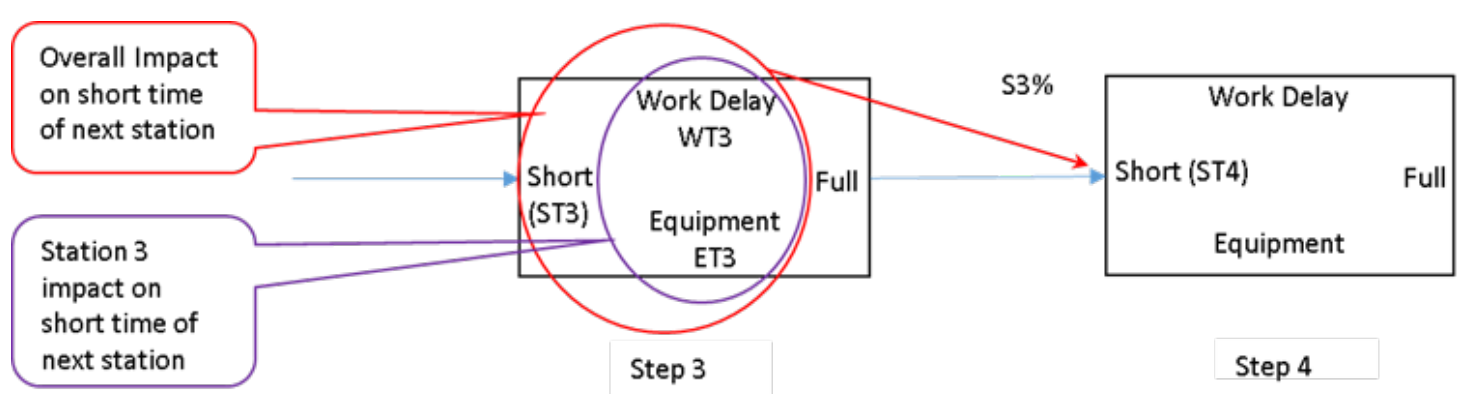

Figure 4. Model to link impact between two steps

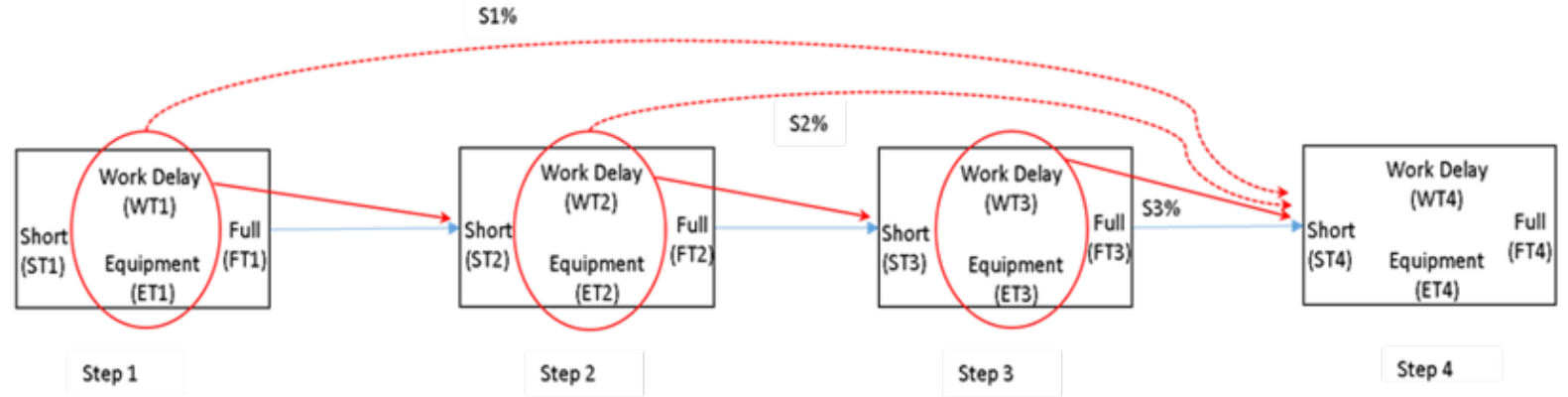

Figure 5. Model to link impact of multiple Steps

The throughput contribution factor of any step in the whole production line, from first step to last step, can be quantified. This evaluation method can be utilized with historical data for productivity improvement as well as real time data for floor management (real time bottleneck visual display). This model can be applied for one production line, as well as one plant or between plants and supplier.

\subsection{Development of New Operational Availability Model to Link the Impact from One Step to Following Step}

The authors have proposed a method to link throughput impact between steps, as shown in Fig. 4. The impact of any step to following step is consisted of two parts: The one is introduced by this step and the one is inherited from previous step. In this model, each impact factor to following step is calculated proportionally. The inherited impact of Step 3 to Step 4 is calculated in Equation (2). The introduced impact from Step 3 to Step 4 is calculated in Equation (3). The impact from each different downtime causes of this step is also can be quantified individually. Equation (4) and (5) show how to calculate equipment downtime impact and work delay impact from Step 3 to Step 4.

$$
\text { Inherited Impact }=\frac{S T 3}{S T 3+W T 3+E T 3} \times S T 4
$$

Where ST3 is Short time to Step 3 from previous steps.

WT3 is work delay at Step 3

ET3 is equipment downtime at Step 3

ST4 is Short time to Step 4 from Step 3

$$
\text { Introduced Impact }=\frac{W T 3+E T 3}{S T 3+W T 3+E T 3} \times S T 4
$$

Introduced Impact due to Equipment $=$

$$
\frac{E T 3}{S T 3+W T 3+E T 3} \times S T 4
$$

Introduced Impact due to Work Delay $=$

$$
\frac{W T 3}{S T 3+W T 3+E T 3} \times S T 4
$$

Based on this model which links impact between steps, the impact of each step to whole system with multiple steps can be developed. The calculation is applicable to large or small scale systems.

\section{Example: Implementation of New Operational Availability Model into Toyota USA}

We have applied for new operational availability model into Toyota in U.S.A. (Toyota USA) with a 4 step system as shown in Fig. 5.

$$
\begin{gathered}
\text { Inherited Impact of Step } 2=\frac{S T 2}{S T 2+W T 2+E T 2} \times \\
\frac{S T 3}{S T 3+W T 3+E T 3} \times S T 4
\end{gathered}
$$

Where $S T 2$ is Short time to Step 2 from previous steps.

$W T 2$ is work delay at Step 2

ET2 is equipment downtime at Step 2

$S T 2$ is Short time to Step 3 from Step 2

$$
\begin{gathered}
\text { Introduced Impact of Station } 2=\frac{W T 2+E T 2}{S T 2+W T 2+E T 2} \times \\
\frac{W T 3+E T 3}{S T 3+W T 3+E T 3} \times S T 4
\end{gathered}
$$

$$
\begin{gathered}
\text { Inherited Impact of Step } 1=\frac{S T 1}{S T 1+W T 1+E T 1} \times \\
\frac{S T 2}{S T 2+W T 2+E T 2} \times \frac{S T 3}{S T 3+W T 3+E T 3} \times S T 4
\end{gathered}
$$


Where $S T 1$ is Short time to Step 1 from previous steps. in Fig.5.

$W T 1$ is work delay at Step 1

ET1 is equipment downtime at Step 1

$S T 1$ is Short time to Step 2 from Step 1

$$
\begin{aligned}
& \text { Introduced Impact of Station } 1=\frac{W T 1+E T 1}{S T 1+W T 1+E T 1} \times \\
& \frac{S T 2}{S T 2+W T 2+E T 2} \times \frac{S T 3}{S T 3+W T 3+E T 3} \times S T 4
\end{aligned}
$$

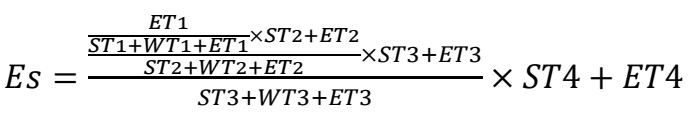

Es is the overall equipment impact to the system shown in Fig.5.

$$
\text { EOA }=\frac{\text { Production Time }-E s}{\text { Production Time }} \times 100 \%
$$

$E O A$ is the Equipment $\mathrm{OA}$ of the overall system shown in Fig.5.

$$
W O A=\frac{\text { Production Time }-W s}{\text { Production Time }} \times 100 \%
$$

\section{Case Study with New Operational Availability Model}

\subsection{Case Study with New Operational Availability Model for Toyota USA}

The new operational availability model was confirmed in the paint shop of Toyota Motor Manufacturing Texas (TMMTX) for Toyota USA, as shown in Fig.6. The target of this study is to improve the shop operational performance and reduce the overtime. There are over 20 steps in this system and we need to fully understand the throughput issue at each step for improvement. The final step was set as study target to judge the shop performance and throughput issues (step in red circle in Fig.6).

$W O A$ is the Work Delay OA of the overall system shown

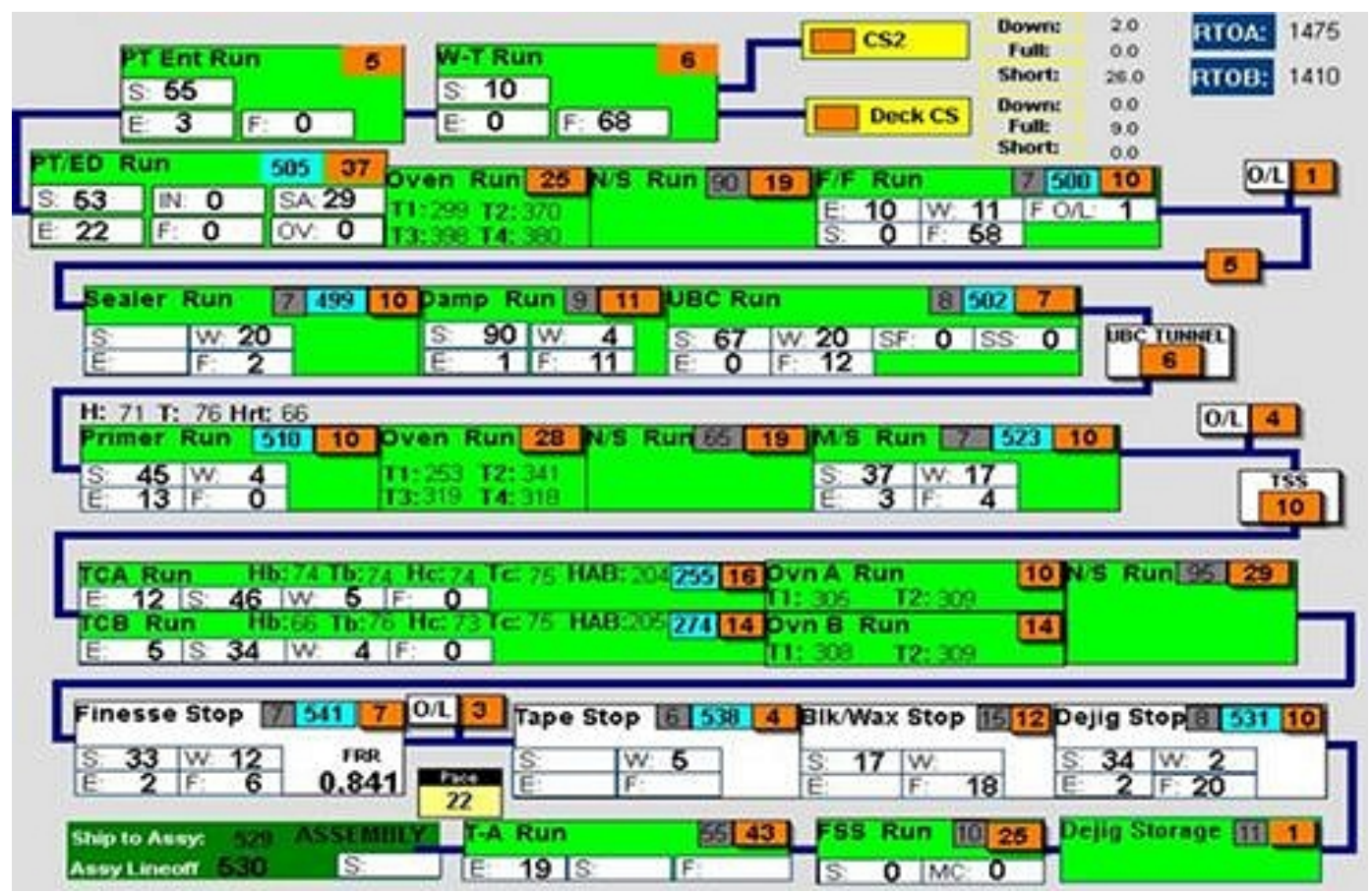

Figure 6. Case study automotive shop layout in TMMTX 
Figure 7 shows the evaluation result with new operational availability model. The short time impact from previous steps is clearly quantified in Fig.7 (a). The 5 steps before the last step are the contributors of the short time. This information helps the management to prioritize the efforts for problem solving and improvement. The impact level from different downtime is clearly identified in Fig.7 (b). The information helps the management to prioritize resource who is responsible for the majority impact to focus on productivity. In this case, the production group is responsible for the work delay and they will lead the improvement activity.

\section{Final Station Short Reason}

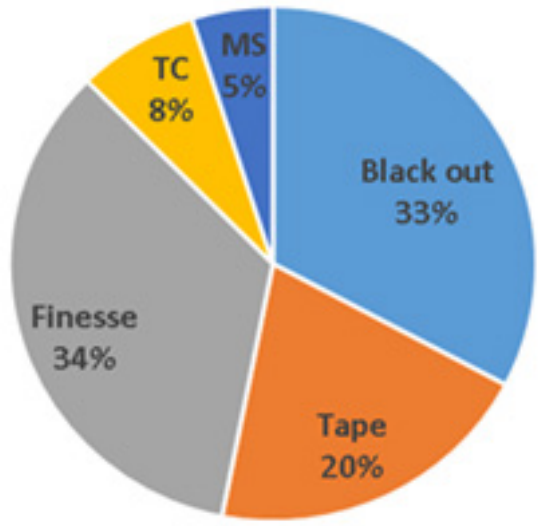

(a)

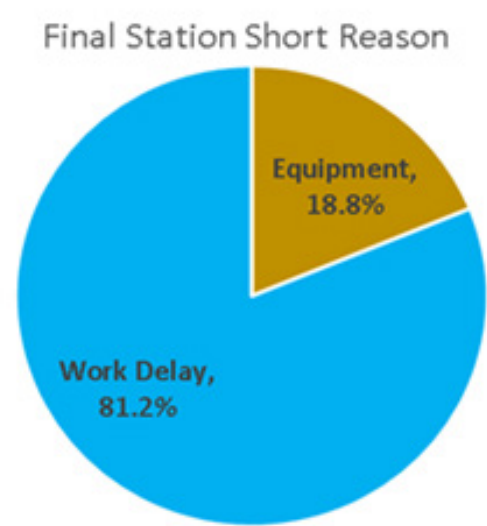

(b)

Figure 7. Final step throughput evaluation result with new operational availability model

\subsection{Effectiveness of New Operational Availability Model}

Figure 8 (a) shows the throughput improvement with problem solving activities at steps with most impact and correct working group. In 6-months period, the shop OA improves $1.7 \%$. This is because the production group who is responsible for the work delay made $1.5 \%$ improvement as shown in Fig.8 (b).

Another benefit of this OA model is that with clarified equipment $\mathrm{OA}$ and work delay OA, the target level can be set individually. The target level for each category can refer to plants with similar set up in Japan or abroad. The target setting will be more feasible and effective. It also helps to control the cost by invest into correct improvement area to achieve the best overall OA target.

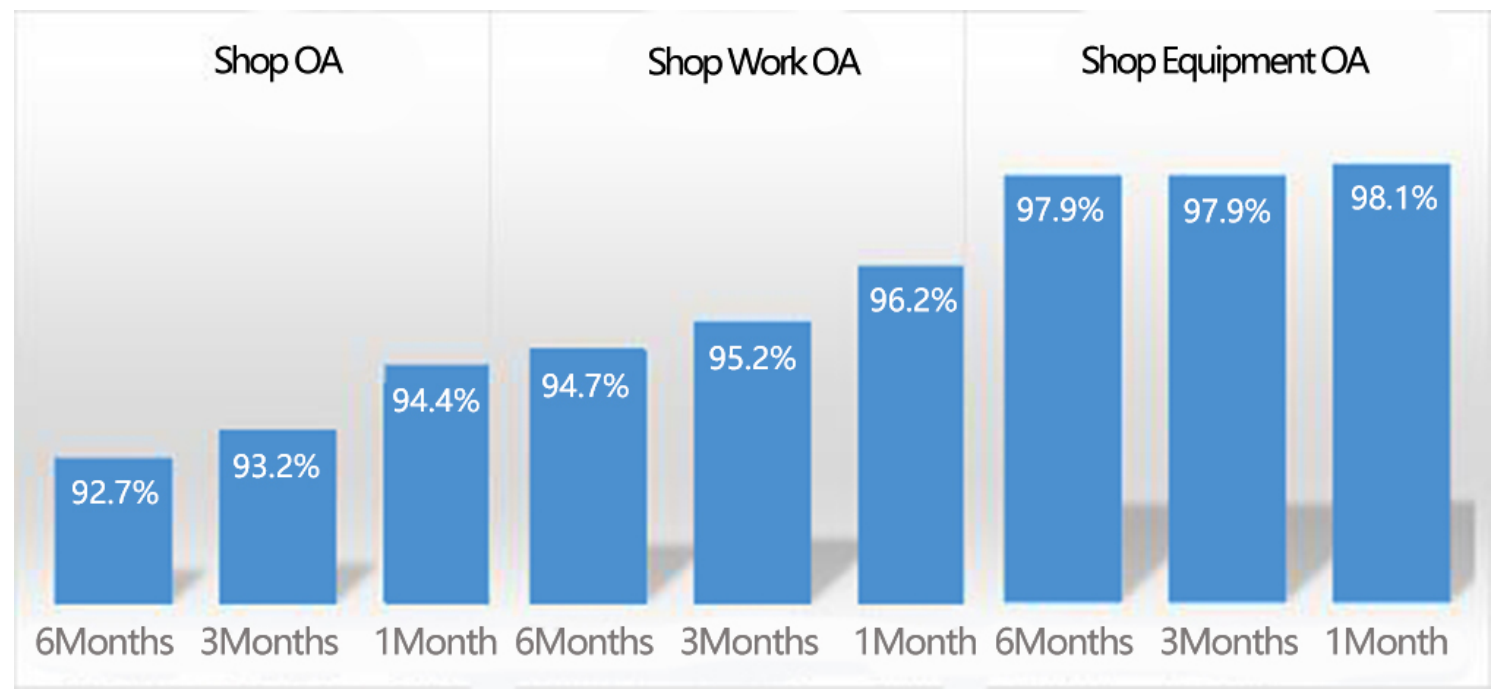

(a)

(b)

(c)

Figure 8. Final step operational availability improvement result 


\section{Conclusions}

A new study method of production throughput: new operational availability model is formulated and analyzed. The throughput impact of each step in TPS production line can be identified by analyzing relationships between the so-called manufacturing blockage (Full) and manufacturing starvation (Short) of each step. This leads to a simple rule for throughput analysis. This study will evaluate the overall effectiveness of the production process. These activities optimize the effectiveness of the TPS. This method can be used to study real-time data or the data over a period of time with historical data. It is a useful indicator for throughput performance tracking and improvement. This evaluation model will standardize the operational availability calculation. A case study at Toyota USA is described. This new operational availability model is also expected to contribute to the extension of "Advanced TPS" concept in real manufacturing environment.

\section{REFERENCES}

[1] Sakai, H. and Amasaka, K., "Demonstrative verification study for the next generation production model: Application of the Advanced Toyota Production System", Journal of Advanced Manufacturing Systems, Vol.7: No.2, 195/219 (2008)

[2] Danny J. Johnson, "A framework for reducing manufacturing throughput time", Journal of Manufacturing Systems, Vol.22: Issue 4, 283/298 (2003)

[3] Kinjal Suthar, Vivek Deshpande, "Review on reducing manufacturing throughput time: Various tools and technologies, Journal of Emerging Technologies and Innovative Research, Vol.1: Issue 6, 542/546 (2014)

[4] Jun Duanmu, Kevin Taaffe, "Measuring manufacturing throughput using takt time analysis and simulation", Proceedings of the 2007 Winter Simulation Conference (2007)

[5] Wallace J. Hopp, Mark L. Spearman, "Throughput of a constant work in process manufacturing line subject to failures", International Journal of Production Research, Vol.29: Issue 3, 635/655 (1991)

[6] Shingo Shigeo, A study of the Toyota Production System: From an industrial engineering viewpoint, Productivity Press, New York (1989)

[7] Ohno Taiichi, Toyota Production System, Diamond Inc. (1978)

[8] Jeffrey K. Liker, The Toyota Way (14 Management Principles from the World's Greatest Manufacturer), McGraw-Hill Education (2003)

[9] Amasaka, K. and Sakai, H., "Availability and reliability information administration system ARIM-BL by methodology in inline-online SQC", International Journal of Reliability, Quality and Safety Engineering, Vol.5: No. 1,
$55 / 63(1998)$

[10] Amasaka, K. and Sakai, H., "Improving the Reliability of Body Assembly Line Equipment", International Journal of Reliability, Quality and Safety Engineering, Vol.3: No. 1, $11 / 24$ (1996)

[11] Sakai, H. and Amasaka, K., "V-MICS, Advanced TPS for strategic production administration", Journal of Advanced Manufacturing Systems, Vol.4: No.6, 5/20 (2005)

[12] Sakai, H. and Amasaka, K., "Strategic HI-POS, intelligence production operating system", WSEAS TRANSACTIONS on ADVANCES in ENGINEERING EDUCATION, Vol. 3: No.3, 223/230 (2006)

[13] Sakai, H. and Amasaka, K., "Human-Integrated Assist Systems for Intelligence Operators", Encyclopedia of Networked and Virtual Organizations, INFORMATION SCIENCE REFERENCE, New York: Hershey, 678/687 (2008)

[14] Sakai, H. and Amasaka, K., "Human Digital Pipeline Method Using Total Linkage Through Design to Manufacturing", Journal of Advanced Manufacturing Systems, Vol.6: No.2, 101/113 (2007) 